\title{
Prevalencia y mortalidad de Enfermedad Renal Crónica en Guatemala (2008-2018)
}

\section{Prevalence and mortality of Chronic Renal Disease in Guatemala (2008-2018)}

\author{
Berta Sam-Colop \\ Vigilancia Epidemiológica de Enfermedades No Transmisibles, Departamento de Epidemiologia, \\ Ministerio de Salud Pública y Asistencia Social, Guatemala
}

*Autor al que se dirige la correspondencia: bsam@mspas.gob.gt

Recibido: 14 de enero 2020 / Revisión: 27 de febrero 2020 / Aceptado: 10 de marzo 2020

\section{Resumen}

T a enfermedad renal crónica (ERC) es una patología que se asocia a la diabetes y enfermedades cardiovasculares

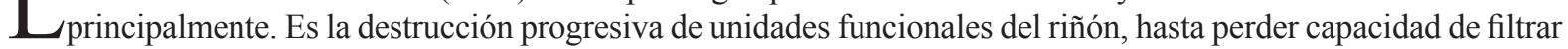
sustancias tóxicas. Con el objetivo de contar con información y conocimientos epidemiológicos oportunos, que permitan contribuir a una adecuada orientación de acciones en salud pública, se llevó a cabo un análisis descriptivo de las bases de datos de morbilidad del Sistema Gerencial de Salud (Sigsa), Unidad Nacional de Atención al Enfermo Renal Crónico (Unaerc), y de mortalidad del Instituto Nacional de Estadística (INE), Códigos CIE10:N18-19. A partir de 2013 se agregaron casos de Unaerc a Sigsa; esto incrementó seis veces el número $(601 / 4,099)$ entre 2012-2013; de igual forma se incrementó entre 2013/2018, 87 \% (4,099/7,760) y entre 2008/2017 se incrementó 15 veces más. En 2018 el mayor número de casos se reportó en Guatemala, Santa Rosa, Petén y Escuintla. Las tasas de prevalencia más altas, fueron en Santa Rosa, Jutiapa, Petén, Guatemala, Escuintla y Quetzaltenango. El grupo de edad con mayor número de casos fue de 25 a 59 años. Entre 2008 y 2017, se documentaron 19,491 casos en estadio V. Durante los últimos 10 años, ha incrementado $56 \%$ la mediana de fallecidos, siendo 2,085 casos anuales. La mortalidad fue mayor en los departamentos con clima cálido, con ocupaciones elementales y sin ninguna escolaridad. Es importante fortalecer el diagnóstico para la detección de estadios tempranos, además asegurar el tratamiento de diabetes mellitus y enfermedades cardiovasculares.

Palabras claves: Salud renal, Prevalencia, Mortalidad, Salud Pública

\section{Abstract}

$\mathrm{C}$ hronic kidney disease (CKD) is a pathology mainly associated with diabetes and cardiovascular diseases. It is the progressive destruction of functional units of the kidney, until losing the ability to filter toxic substances. In order to have timely epidemiological information and knowledge, timely to contribute with an adequate orientation of actions in public health, a descriptive analysis was carried out of databases of Managerial Health System (Sigsa), National Unit for Chronic Kidney Disease Attention (Unaerc) for morbidity, and the National Institute for Statistics (INE) for mortality, ICD10 codes: N181. As of 2013, Unaerc cases were added to Sigsa; this increased the number $(601 / 4,099)$ six times between 2012-2013. Similarly, it increased between 2013-2018, $87 \%(4,099 / 7,760)$ and between 2008/2017 it increased 15 times more. In 2018 the highest number of cases was reported in Guatemala, Santa Rosa, Peten and Escuintla. The highest prevalence rates were in the departments of Santa Rosa, Jutiapa, Peten, Guatemala, Escuintla and Quetzaltenango. The age group with the highest number of cases was 25 to 59 years. Between 2008 and 2017, 19,491 cases were documented on stage V. During the last 10 years, the median number of deaths has increased by 5,685 per year. Mortality was higher in the departments with warm weather, with elementary occupations and without any schooling. It is important to strengthen the diagnosis for the detection of early stages, in order to allow safe treatment for diabetes mellitus and cardiovascular diseases.

Keywords: Kidney health, prevalence, mortality, Public health 


\section{Introducción}

La Enfermedad Renal Crónica (ERC), se asocia con otras enfermedades crónicas tales como diabetes, hipertensión y enfermedad cardiovascular. La Sociedad Chilena de Nefrología indica que es la destrucción progresiva de las unidades funcionales del riñón hasta que no pueda realizar todas las funciones requeridas, el daño puede darse en uno o ambos riñones, hasta que se pierde la capacidad de filtrar las sustancias tóxicas de la sangre (Martínez-Castelao et al., 2014).

Los pacientes con estadios tempranos de ERC, generalmente no son diagnosticados ni tratados oportunamente $\mathrm{y}$, con frecuencia, presentan múltiples factores de riesgo concurrentes que incrementan el riesgo para pérdida de la función renal, el desarrollo de complicaciones y la muerte cardiovascular precoz.

Los factores de riesgo pueden ser divididos en: factores predisponentes o de susceptibilidad, factores iniciadores y factores perpetuadores del daño renal y su progresión, con algunas combinaciones entre ellos (Bermúdez et al., 2019). Los predisponentes son características de los sujetos que incrementan la probabilidad de desarrollar ERC. Los iniciadores son aquellos que, de manera independiente, pueden ser asociados al desarrollo de ERC, y los de progresión o perpetuadores son aquellos que pueden estar asociados con progresión del daño renal (Tabla 1).

Estos factores, generalmente interactúan a manera de círculo vicioso sobre la función del riñón y provocan la pérdida progresiva de la reserva renal. La identificación de los factores de susceptibilidad y de inicio es importantes para reconocer a las personas con mayor riesgo de desarrollar ERC, mientras que la identificación de los factores de progresión son útiles para definir cuáles de esos pacientes con ERC tienen mayor riesgo de progresar hasta las etapas finales de la enfermedad (Flores, 2010).

La enfermedad renal crónica de causa no tradicional (ERCnT) es una patología de la que en los últimos años han surgido numerosos casos entre los trabajadores agrícolas, así como entre otros que realizan trabajo manual en diversas regiones del mundo (Bermudez et al., 2019; Lozier et al., 2016; Wegman, 2017). La enfermedad no parece deberse a las causas clásicas de la enfermedad renal (por ejemplo, diabetes, hipertensión y enfermedad glomerular) (Canal et al., 2014; Gorostidi et al., 2014) ).

En el modelo multideterminante se consideran dos mecanismos hipotéticos principales en la etiología de esta patología. Por un lado, las prácticas agrícolas que involucran un trabajo intenso y el estrés térmico agravado por un evidente cambio climático, son las principales causas de esta disfunción renal, en la que los cortadores de caña son considerados una población centinela ocupacional (Wesseling et al., 2020). Y por el otro, además de las condiciones laborales precarias y extremas, la exposición a agroquímicos y antiinflamatorios no esteroidales, en un contexto de vulnerabilidad social y clima tropical, han determinado que esta patología se constituya en un importante problema de salud pública en el país (Johnson et al., 2019; Lozier et al., 2016).

El objetivo de esta exploración, es contar con información y conocimientos epidemiológicos oportunos, que permita tener el conocimiento de la magnitud y causas de la ERC, con el fin de contribuir a una adecuada orientación de las acciones en salud pública.

Los datos se obtuvieron del análisis descriptivo de bases de datos de morbilidad del Sistema Gerencial de Salud (Sigsa),de la Unidad Nacional de Atención al Enfermo Renal Crónico (Unaerc) y de mortalidad de Instituto Nacional de Estadística (INE), utilizando los códigos CIE10: N18-19. Se calcularon las tasas, percentiles y medidas de tendencia central.

\section{Contenido}

\section{Prevalencia}

El número de casos prevalentes por ERC, entre 2008 y 2012 con datos de Sigsa, son menores a los años 2013-2018, esto debido que, a partir de 2013 se agregaron los casos prevalentes de Unaerc a la base de datos de Sigsa; lo que incrementó más de seis veces el número de casos $(601 / 4,099)$ entre 2012 y 2013; de igual forma se documentó un incremento de $87 \%$ $(4,099 / 7,660)$ de casos entre 2013 comparado con 2018. (Figura 1).

Se ha documentado 35,877 casos prevalentes de ERC, por la red de servicios del Ministerio de Salud Públicas (MSPAS) y Unaerc de 2008 a 2018. Con datos de mortalidad según INE se documentaron 19,491 casos, de donde se puede decir que la tasa de letalidad es de $54 \%$ (Figura1).

En Guatemala durante los últimos 10 años (20082018) con estos datos (MSPAS y Unaerc) se considera que ha incrementado 15 veces más la prevalencia; en Unaerc se presta servicio a una mediana de más de 5,000 pacientes anuales (Figura 1). 
Tabla 1

Factores de riesgo para el desarrollo de enfermedad renal crónica

\begin{tabular}{|c|c|c|}
\hline Predisponentes & Iniciadores & Perpetuadores \\
\hline $\begin{array}{l}\text { Edad avanzada (>60 años) } \\
\text { Historia familiar de ERC } \\
\text { en el grupo étnico } \\
\text { (origen hispano) } \\
\text { Género masculino } \\
\text { Síndrome metabólico } \\
\text { Reducción de la masa } \\
\text { renal Bajo nivel } \\
\text { socioeconómico y de } \\
\text { educación }\end{array}$ & $\begin{array}{l}\text { Enfermedades renales } \\
\text { primarias } \\
\text { Diabetes mellitus } \\
\text { Hipertensión arterial } \\
\text { sistémica } \\
\text { Enfermedades } \\
\text { autoinmunes } \\
\text { Nefrotoxinas } \\
\text { Aines } \\
\text { Aminoglucósidos } \\
\text { Medio de contraste } \\
\text { IV } \\
\text { Otros } \\
\text { Patologías urológicas } \\
\text { Obstrucción } \\
\text { urinaria } \\
\text { Litiasis urinaria } \\
\text { Infección urinaria } \\
\text { recurrente } \\
\text { Enfermedades hereditarias }\end{array}$ & $\begin{array}{l}\text { Proteinuria } \\
\text { TA sistólico }>130 \mathrm{mmHg} \\
\text { Alta ingesta de proteínas } \\
\text { Pobre control de la glucemia } \\
\text { Obesidad } \\
\text { Anemia } \\
\text { Dislipidemia } \\
\text { Tabaquismo } \\
\text { Hiperuricemia } \\
\text { Nefrotoxinas } \\
\text { Enfermedad cardiovascular }\end{array}$ \\
\hline
\end{tabular}

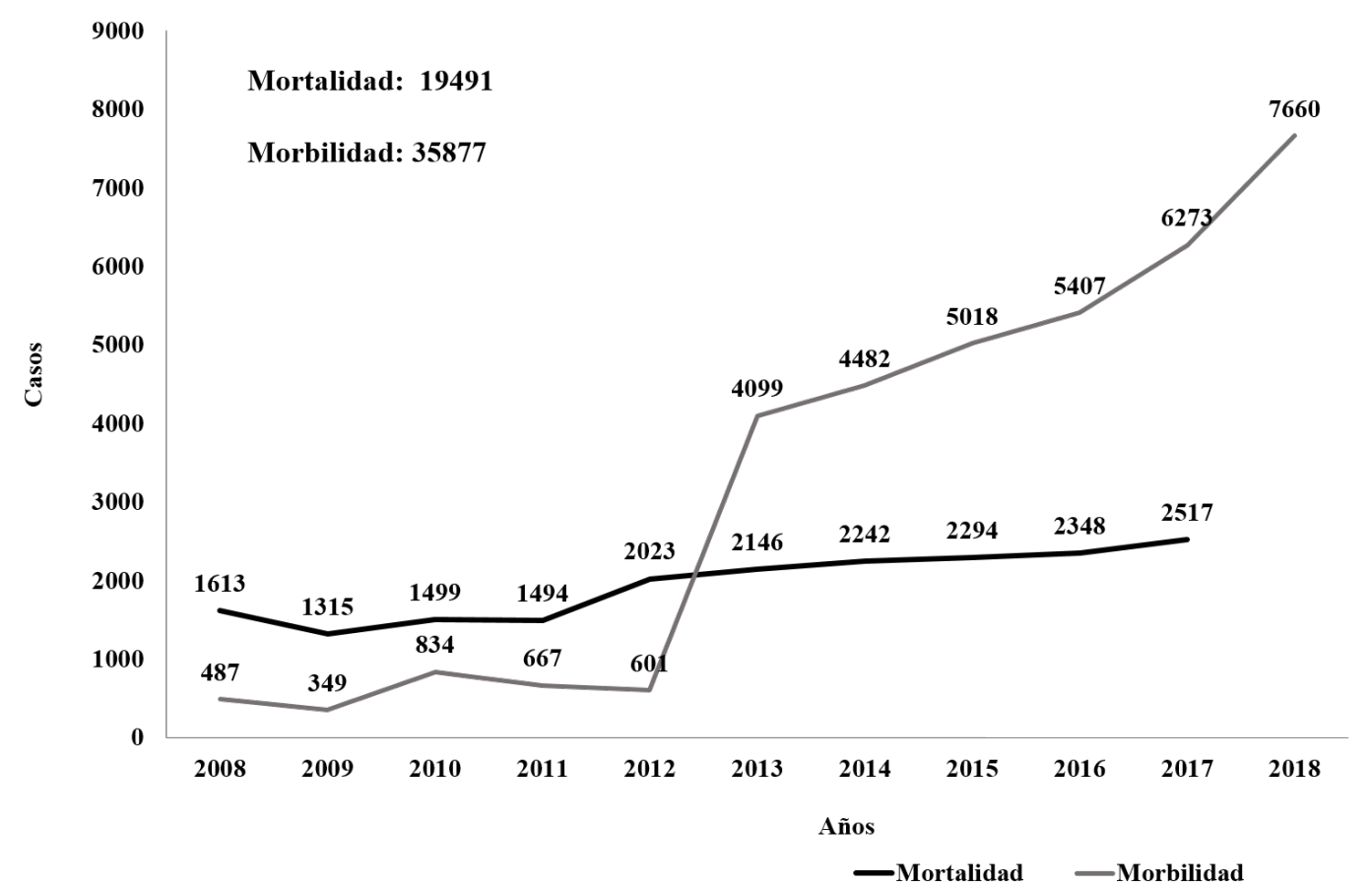

Figura 1. Casos de ERC, por morbilidad y mortalidad. Guatemala 2008-2018. Fuente: INE/SIGSA/UNAERC (*a julio 2018)/Departamento Epidemiología Ministerio de Salud Pública y Asistencia Social, Guatemala. 
La distribución de casos por departamento muestra que para 2018 los departamentos con mayor número de casos son: Guatemala, seguido por Santa Rosa, Petén y Escuintla. Las tasas más altas, las encontramos en los departamentos de Santa Rosa, Jutiapa, Petén, Guatemala y Escuintla (Tabla 2). En esta serie de años, observamos frecuentemente mayor riesgo estimado por tasa de prevalencia en Santa Rosa, Suchitepéquez, Jutiapa, Escuintla, Guatemala, Petén y Quetzaltenango.

Los departamentos con menor riesgo, en el periodo 2008-2018, estimado por tasas son: Alta Verapaz, Totonicapán, Huehuetenango, Quiche y Sololá. Esto posiblemente explicado por la falta de centros especializados para la detección de enfermedades renales crónicas, además por el factor de la temperatura media que no sobrepasa los $16^{\circ} \mathrm{C}$.

Por distribución de tasas de prevalencia, por grupo de edad, en los últimos tres años, encontramos que el grupo de mayores de 60 años muestra las tasas más altas. En 2017 en el segundo grupo de barras, donde se suman los datos de MSPAS y Unaerc, observamos incremento de tasas en todos los grupos de edad, siendo mucho más altas en mayores de 60 años; mientras que el grupo de barras de datos del MSPAS, se observa leve incremento entre año y año (Figura 2).

Por número de casos de ERC, el comportamiento es diferente; el número es más alto en el grupo de 20-59 años, comparado con los otros grupos de edad en los últimos tres años, mostrando que la carga de atención en la red de servicios de salud, es en la población económicamente activa (Figura 3).

\section{Mortalidad}

Las tasas de mortalidad por ERC, entre 2008 y 2017 con datos de INE, observamos la distribución por departamento para 2017, los departamentos con mayor tasa son Retalhuleu, Suchitepéquez, Escuintla, Santa Rosa y Guatemala. Este comportamiento se mantiene en toda la serie de años, siendo los mismos departamentos los de mayor riesgo. El departamento con mayor número de casos en el periodo 2008-2017

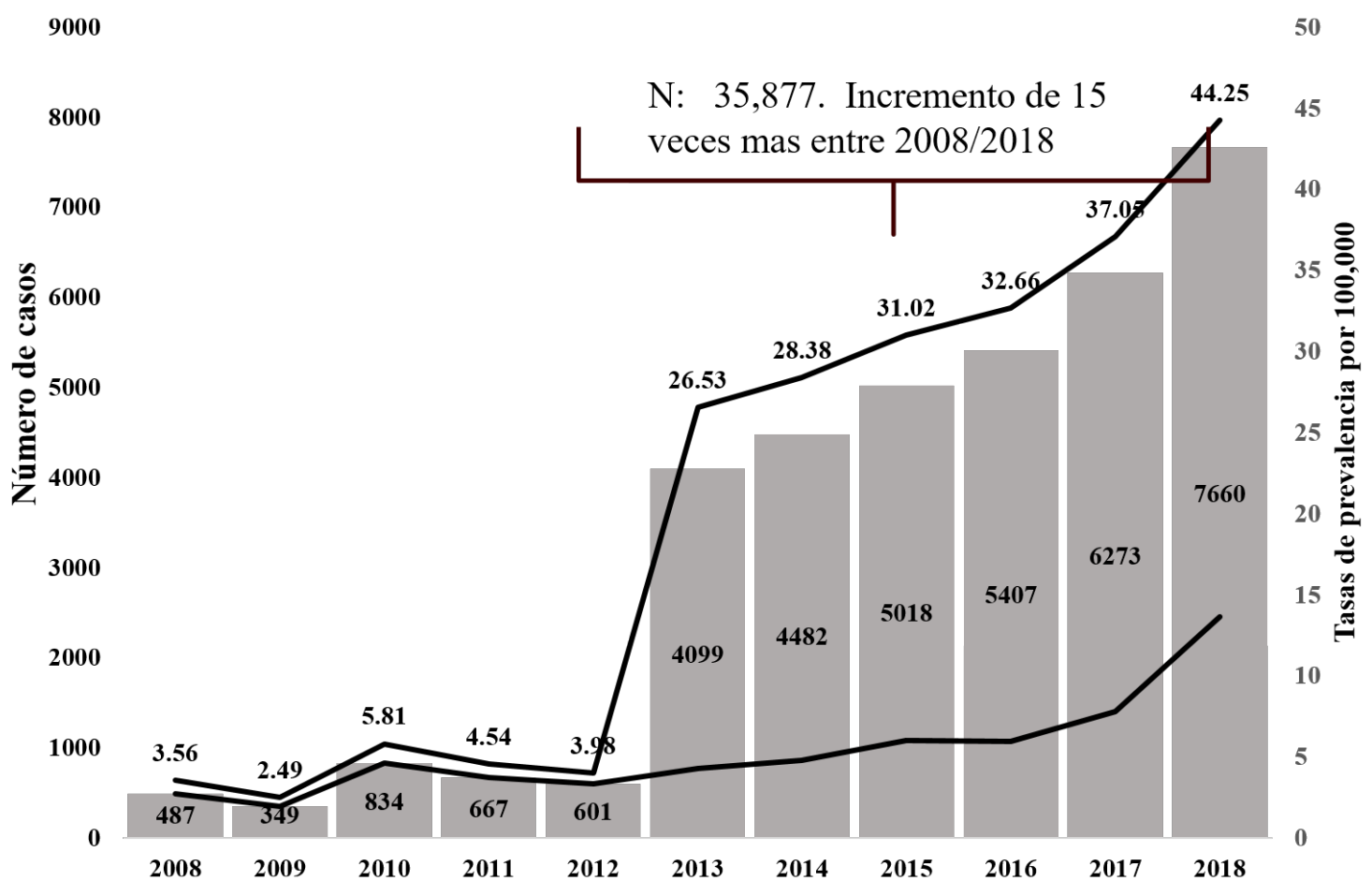

Casos MSPAS/UNAERC -Casos red MSPAS -Prevalencia

Figura 2. Casos y tasas de prevalencia de ERC por grupo edad. Guatemala 2008-2018. Fuente: SIGSA 2008-2018/UNAERC 2013-2018* (*enero julio)/Departamento Epidemiología Ministerio de Salud Pública y Asistencia Social, Guatemala. 


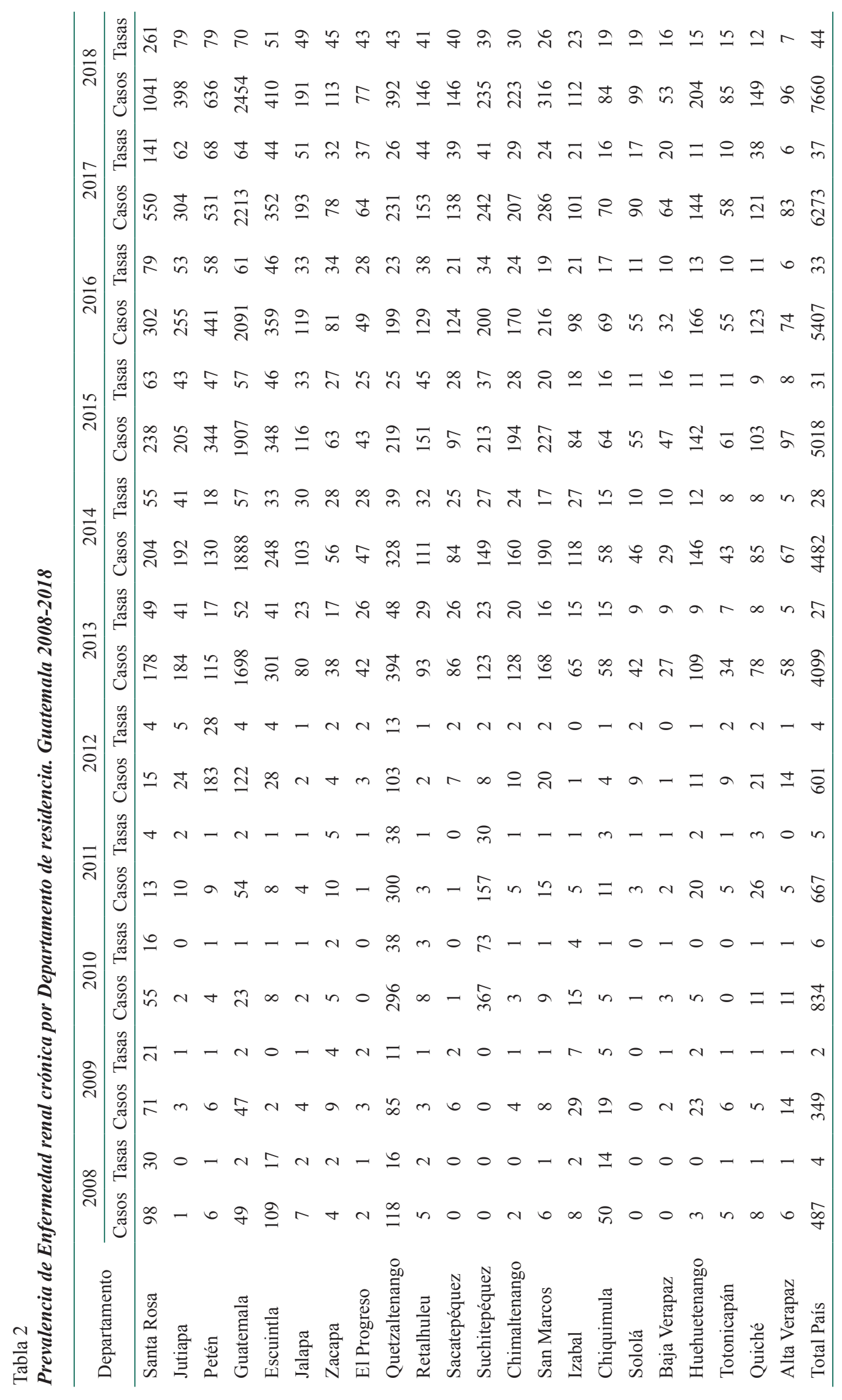




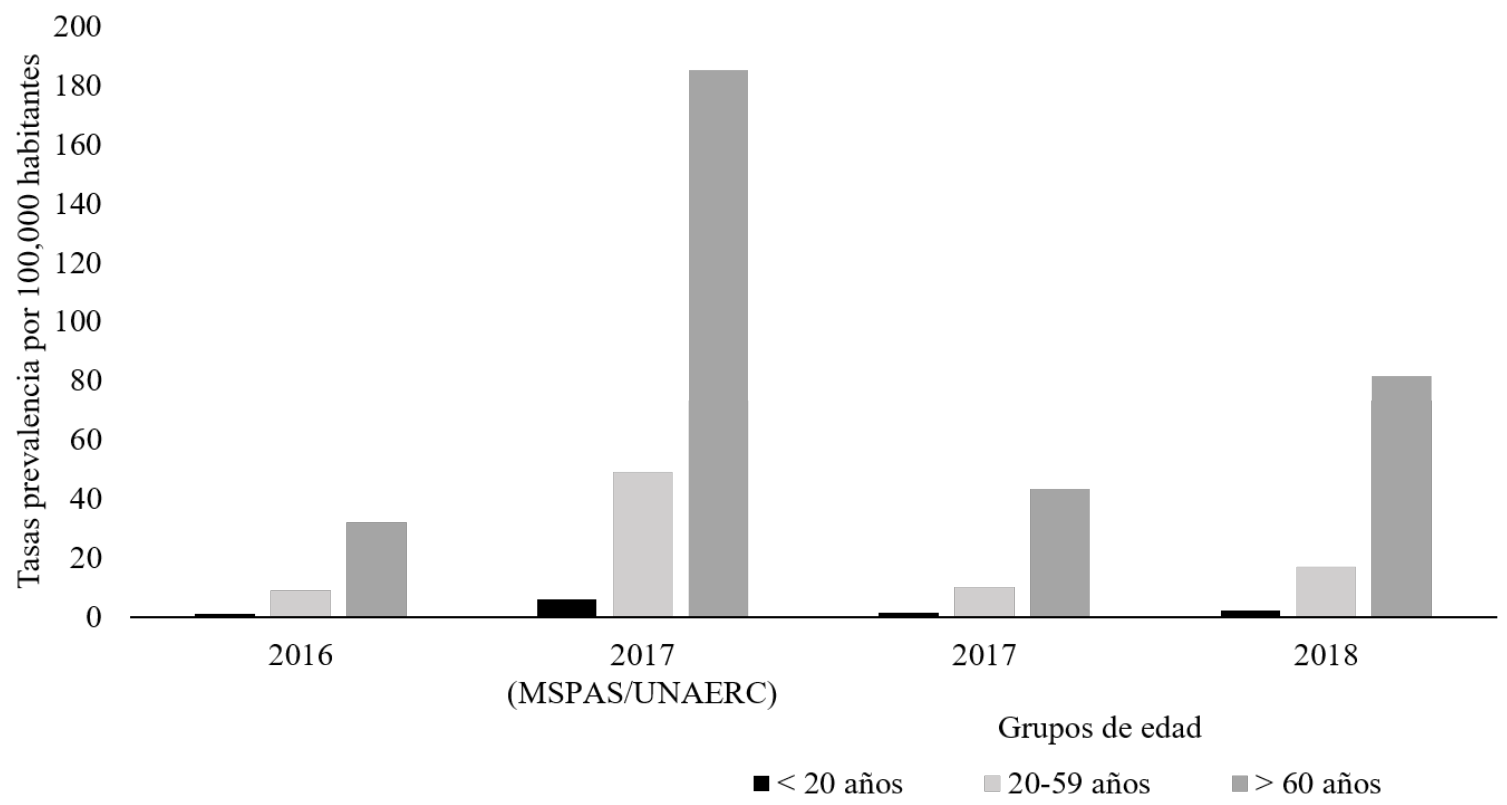

Figura 3. Casos de ERC por grupo de edad. Guatemala 2016-2018. Fuente: SIGSA/UNAERC/Departamento Epidemiología Ministerio de Salud Pública y Asistencia Social, Guatemala.
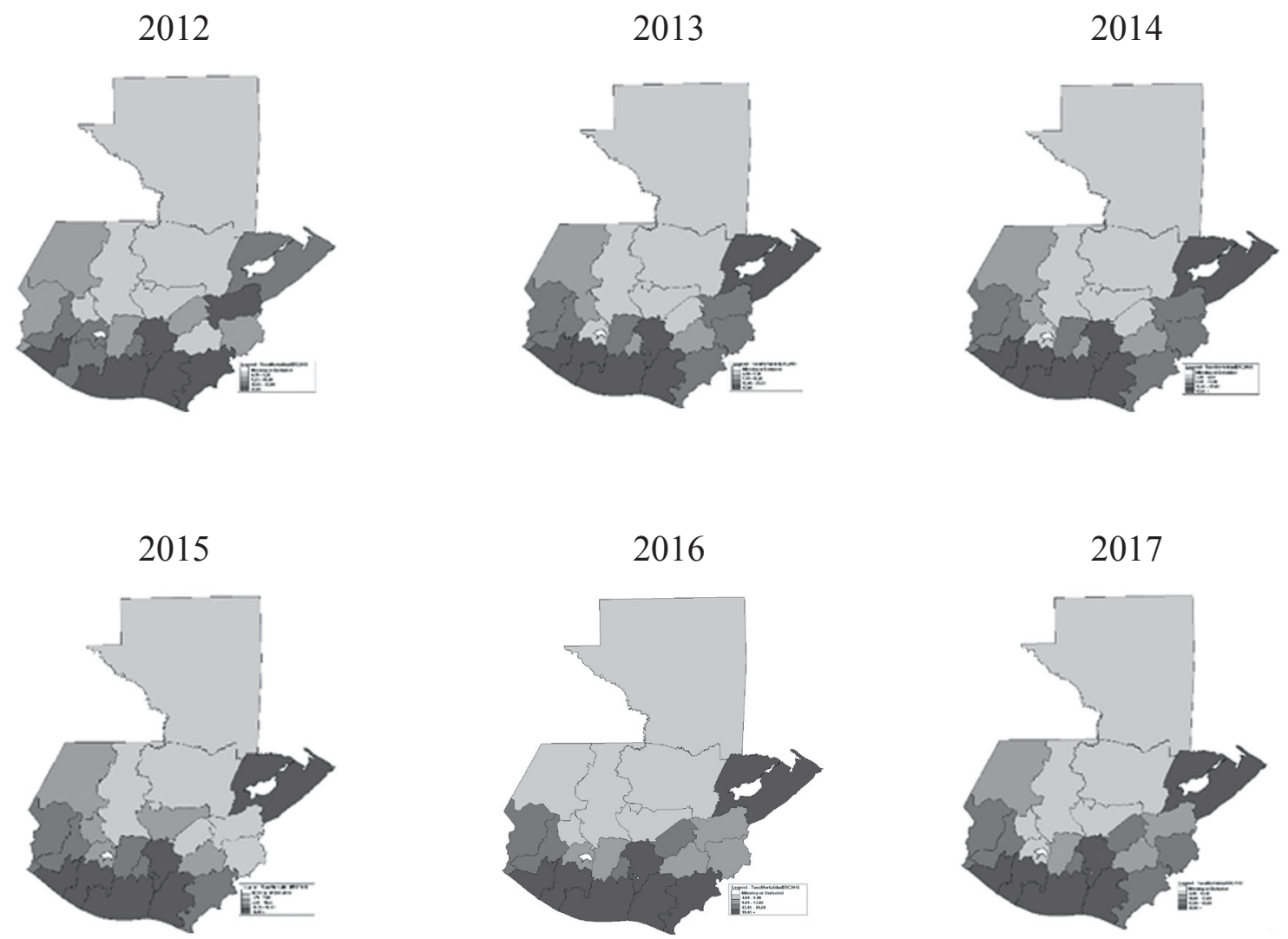

Figura 4. Mapa de tasas de mortalidad por enfermedad renal crónica por cuartiles. Guatemala 2012-2017 
es Guatemala. Los departamentos con tasas por abajo del promedio nacional son: Alta Verapaz, Totonicapán, Peten, Baja Verapaz, Quiche, Sololá (Tabla 3; Figura 4).

En el periodo 2008-2017, se documentaron por mortalidad 19,491 casos; estos mediante la variable de lugar de residencia. Encontramos en los mapas de los últimos seis años, que los departamentos ubicados en el cuarto cuartil, los encontramos en la costa del Pacífico, sin embargo, en la costa del Atlántico, también se encuentra el departamento de Izabal en cuarto cuartil, en los últimos cuatro años (Figura 4).

Las tasas de mortalidad comparado entre 2017 con 2008 , ha incrementado $56 \%$, la mediana de casos fallecidos es 2,085 anuales (Figura 5).

\section{Ocupación}

La clasificación que se presenta, obedece a la resolución sobre actualización de la Clasificación Internacional Uniforme de Ocupaciones (CIUO), del Consejo de Administración de la Organización Internacional del Trabajo (OIT) de octubre 2007. Aquí observamos que el mayor porcentaje entre 2010, 2012, 2017, por ocupación, se reportó en actividades elementales, entre ellos encontramos: Limpiadores y asistentes, peones agropecuarios, pesqueros y forestales, peones de la minería, la construcción, la industria manufacturera y el transporte, ayudantes de preparación de alimentos, vendedores ambulantes de servicios y afines, recolectores de desechos y otras ocupaciones elementales; seguido por agricultores, trabajadores calificados agropecuarios, forestales y pesqueros, de oficiales operarios, artesanos de artes mecánicas y otros oficios. Las ocupaciones con menor proporción son: profesionales, personal de apoyo administrativo, técnicos profesionales y ocupaciones militares (Figura 6).

\section{Escolaridad}

En estas tres observaciones (2010, 2012, 2017), la proporción más alta se encuentra entre los que no asistieron a un establecimiento educativo, seguido de nivel primario y desconocido. Sin embargo, en 2017, se observa un porcentaje mínimo en nivel desconocido (Figura 7).

\section{Discusión}

Las estrategias para mejorar el panorama de la ERC requieren de la identificación de los factores de riesgo para daño renal, lo que permitirá orientar los esfuerzos para el diagnóstico temprano, en las poblaciones con alto riesgo de desarrollarla y, subsecuentemente, permitirá la aplicación oportuna de intervenciones de nefroprotección para prevenir o retardar la progresión del daño renal

- La identificación de los factores de riesgo supone las siguientes ventajas:

- Identificar a los sujetos con riesgo más alto de desarrollar ERC.

- Clasificar los factores de riesgo en predisponentes, iniciadores y perpetuadores de ERC.

- Identificar factores de riesgo que son modificables o no modificables y que pueden intervenir en la progresión del daño renal y asociarse muerte cardiovascular precoz y de otro tipo e insuficiencia renal crónica terminal (IRCT).

- Alertar a la población con factores de riesgo, acerca de los efectos negativos que tienen los patrones inapropiados de estilo de vida y dieta, así como el uso de nefrotóxicos.

- Facilitar la educación del paciente, para fomentar el autocuidado.

- Aplicar medidas de nefroprotección en fases más tempranas de la enfermedad, para contrarrestar su efecto, con el fin de prevenir, detener o retardar la progresión del daño renal y mejorar la mayoría de las complicaciones de una función renal disminuida.

La enfermedad está fuertemente asociada con trabajar y vivir en un ambiente caluroso, pero aún se desconoce si la causa es una substancia tóxica, un agente infeccioso, una lesión asociada al calor o una combinación de factores (Escamilla-Cejudo et al., 2016; Jayasumana et al., 2017; Lozier et al., 2016).

\section{Conclusiones}

En prevalencia, la ERC presenta un incremento importante por carga de enfermedad en servicios hospitalarios, se identifican alrededor de 5,000 casos incidentes anuales. Entre los departamentos con mayor prevalencia encontramos a Santa Rosa, Guatemala, Petén y Jutiapa. Los grupos de edad más comprometidos por número de casos son los de 20 a 59 años, los adultos presentan más riesgo por tasas.

En el momento del diagnóstico, la ERC suele estar avanzada, desafortunadamente, no siempre hay 


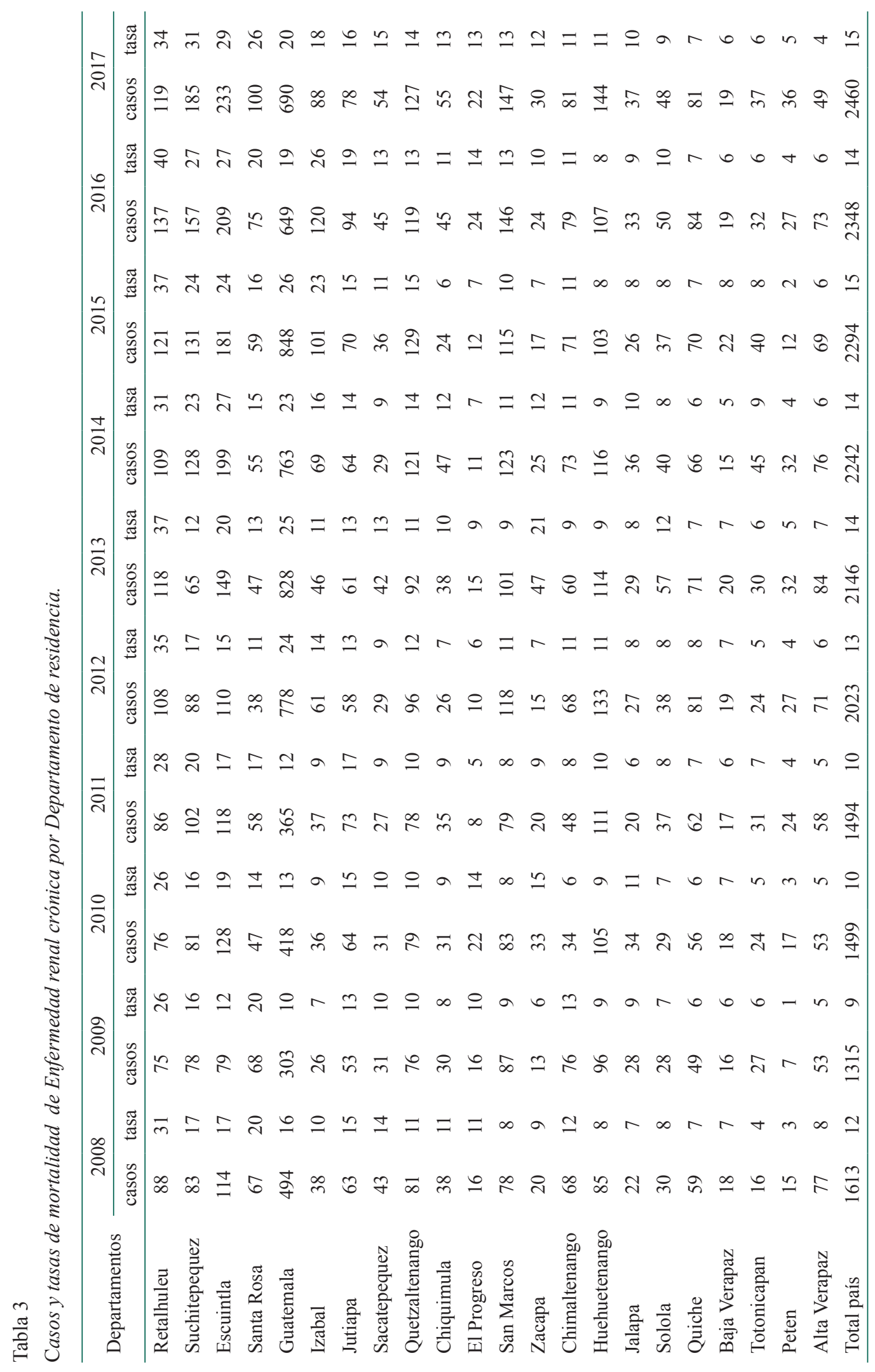




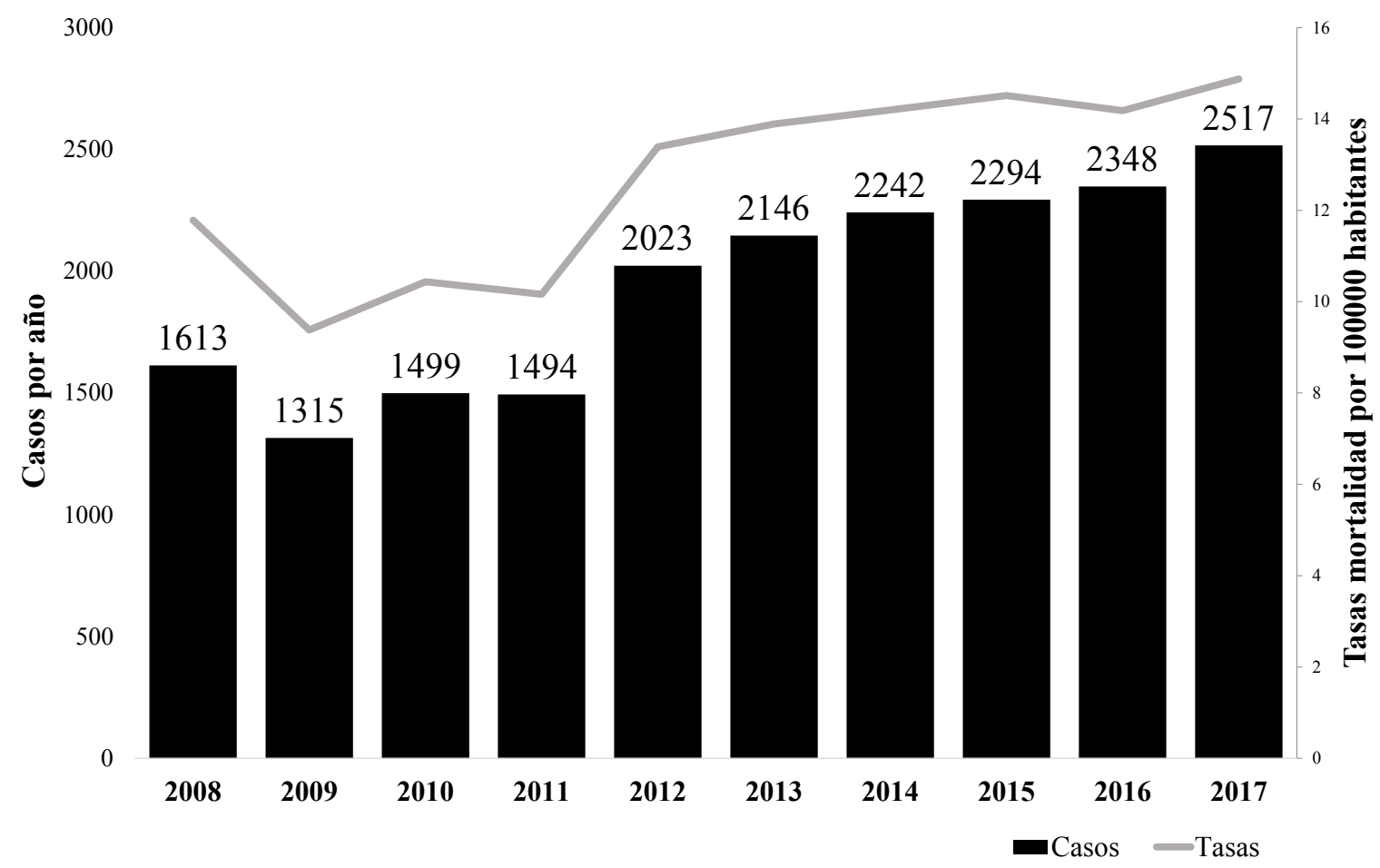

Figura 5. Casos y tasas mortalidad ERC por año. Fuente: INE/Departamento Epidemiología Ministerio de Salud Pública y Asistencia Social, Guatemala.

programas de diálisis disponible. En Guatemala, se presenta en departamentos con clima cálido, en personas con ocupaciones elementales y baja escolaridad (desigualdades socioeconómicas).

Aún no disponemos de datos que permitan un completo análisis de los factores de riesgo, pero se está avanzando en el Registro Guatemalteco de Diálisis y Trasplante Renal, con todos los prestadores de servicios, tantos públicos, del Instituto Guatemalteco de Seguridad Social (Igss), Asociación Guatemalteca de Nefrólogos (AGN) y nefrólogos privados; de igual forma en la vigilancia centinela de ERC en cuatro hospitales de la red nacional del Ministerio de Salud Pública y Asistencia Social (MSPAS), en Escuintla, Petén, Chimaltenango y Quetzaltenango. A partir de 2020, se tendrá mayor información de lo que sucede en el país.

Se recomienda fortalecer el diagnóstico para detección oportuna de estadios tempranos, en personas con el perfil descrito, que realizan labores en la indus- tria agrícola, en zonas con clima cálido y en personas que migran internamente por labores agrícolas temporales , permitiendo identificación oportuna. Así mismo, asegurar el diagnóstico y tratamiento oportuno de factores iniciadores entre ellos: diabetes mellitus y enfermedades cardiovasculares; así como evitar los factores de riesgo predisponentes, como obesidad; y perpetuadores, en la red de servicios de salud del MSPAS, seguro social y otros; para contener la epidemia e identificar oportunamente la ERCnT.

Finalmente se recomienda implementar programas de prevención, control oportuno de ERC tanto en Salud Pública, Igss, y en las empresas agrícolas, así como de vigilancia centinela comunitaria, para responder a las necesidades de salud de la población en general. 


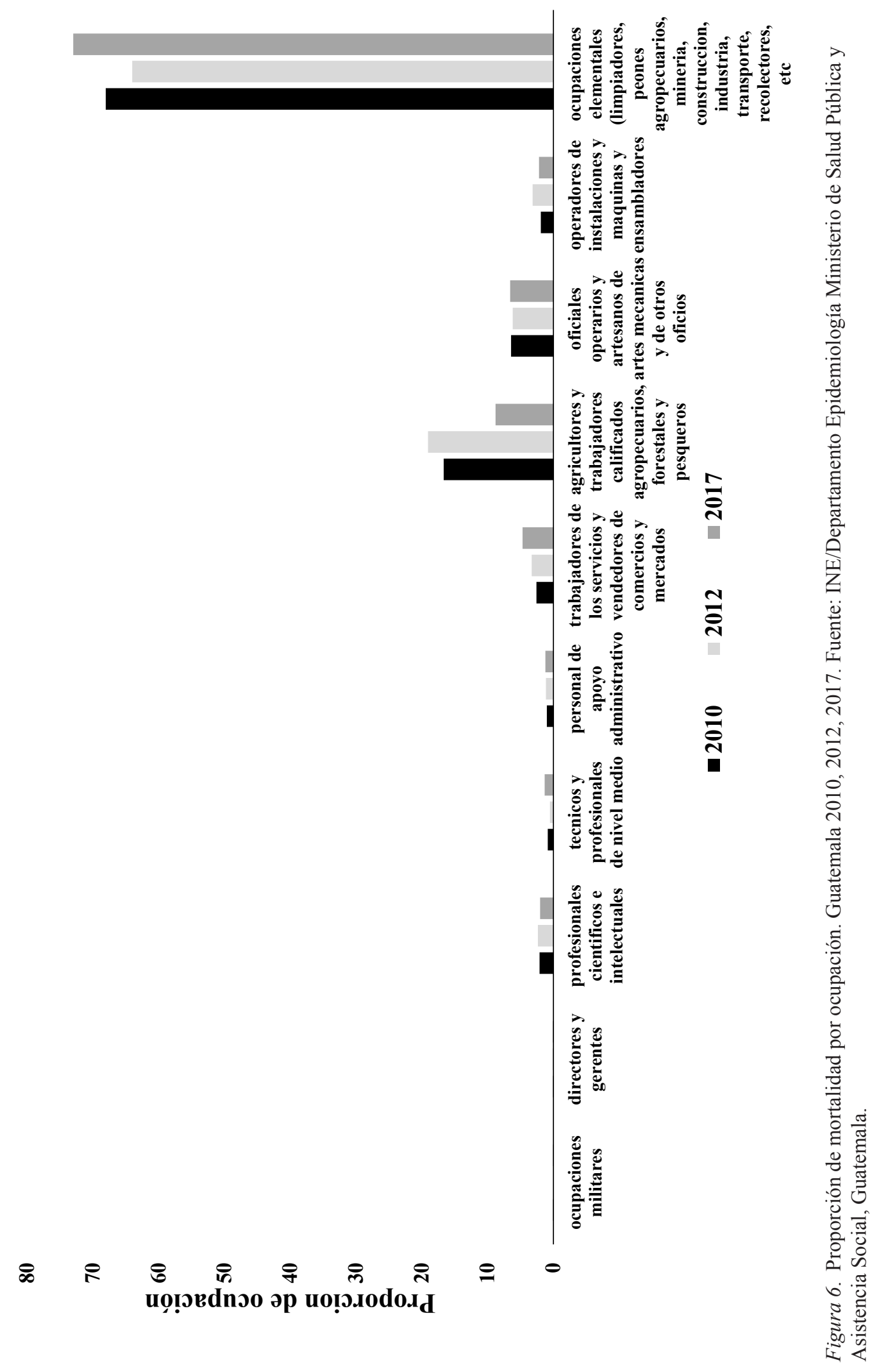


60

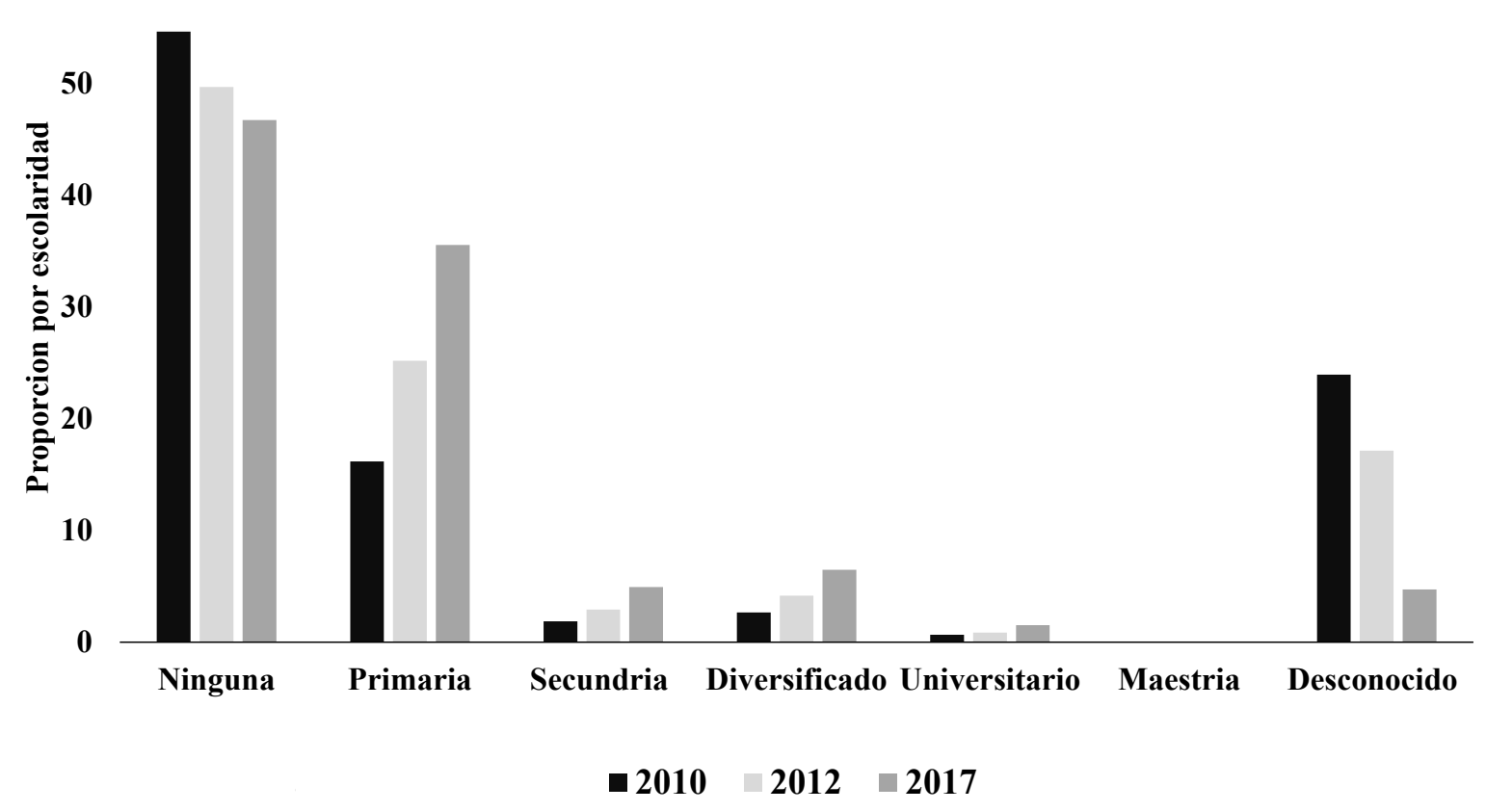

Figura 7. Proporción de mortalidad por escolaridad. Guatemala 2010, 2012 2017. Fuente: INE/Departamento Epidemiología Ministerio de Salud Pública y Asistencia Social, Guatemala.

\section{Agradecimientos}

La autora agradece a, las autoridades del Departamento de Epidemiología del Ministerio de Salud Pública y Asistencia Social, y de Unaerc.

\section{Referencias}

Bermudez, K., Orantes, C., León, M., Jiménez, M., Vidal, N., \& de Vos, P. (2019). Meso-American nephropathy in El Salvador: Increasing farmer participation in MeN prevention efforts. Occupational Diseases and Environmental Medicine, 07(02), 50-63. https://doi.org/10.4236/ odem.2019.72005

Canal, C., Pellicer, R., Facundo, C., Gràcia-García, S., Montañés-Bermúdez, R., Ruiz-García, C., ... Bover, J. (2014). Tablas para la estimación del filtrado glomerular mediante la nueva ecuación CKD-EPI a partir de la concentración de creatinina sérica. Nefrología, 34(2), 223-229.
https://doi.org/10.3265/Nefrologia.pre2013. Dec. 12226

Escamilla-Cejudo, J. A., Báez, J. L., Peña, R., Luna, P. L. R., \& Ordunez, P. (2016). Optimización del registro de muerte por enfermedad renal crónica en las comunidades agrícolas de América Central. Revista Panamericana de Salud Pública, 40(5), 285-293.

Favela, E. A., Gutierrez J. L., Medina, M. C., Rolón, M. L., Sierra C. C., Viniegra, A. (2014). Diagnóstico y tratamiento de la Hipertensión arterial en el primer nivel de atención. México: Instituto Mexicano del Seguro Social (Imss).

Flores, J. C. (2010). Enfermedad renal crónica: epidemiología y factores de riesgo. Revista Médica Clínica Las Condes, 21(4), 502-507. https://doi.org/10.1016/s0716-8640(10)70565-4

Gorostidi, M., Santamaría, R., Alcázar, R., FernándezFresnedo, G., Galcerán, J. M., Goicoechea, M., ... Ruilope, L. M. (2014). Documento de la sociedad española de nefrología sobre las guías 
KDIGO para la evaluación y el tratamiento de la enfermedad renal crónica. Nefrología, 34(3), 302316. https://doi.org/10.3265/Nefrologia.pre2014. Feb. 12464

Jayasumana, C., Orantes, C., Herrera, R., Almaguer, M., Lopez, L., Silva, L. C., \& de Broe, M. E. (2017). Chronic interstitial nephritis in agricultural communities: A worldwide epidemic with social, occupational and environmental determinants. Nephrology Dialysis Transplantation, 32(2), 234241. https://doi.org/10.103/ndt/gfw346

Johnson, R. J., Wesseling, C., \& Newman, L. S. (2019). Chronic kidney disease of unknown cause in agricultural communities. New England Journal of Medicine, 380(19), 1843-1852. https://doi. org/10.1056/NEJMra1813869

Lozier, M., Turcios-Ruiz, R. M., Noonan, G., \& Ordunez, P. (2016). Chronic kidney disease of nontraditional etiology in Central America: A provisional epidemiologic case definition for surveillance and epidemiologic studies. Pan American Journal of Public Health, 40(5), 294300.
Martínez-Castelao, A., Górriz-Teruel, J. L., Bover-San Juan, J., Segura-de la Morena, J., Cebollada, J., Escalada, J., ... Tranche, S. (2014). Documento de consenso para la detección y manejo de la enfermedad renal crónica. Nefrología, 34(2), 243262. https://doi.org/10.32.65/Nefrologia.pre2014. Feb.12455

Wegman, D. (2017). Nefropatía Mesoamericana: Informe del Segundo Taller Internacional de Investigación sobre Nefropatía Mesoamericana. San José CR: Saltra/IRET-UNA.

Wesseling, C., Glaser, J., Rodríguez-Guzmán, J., Weiss, I., Lucas, R., Peraza, S., ... Jakobsson, K. (2020) Chronic kidney disease of non-traditional origin in Mesoamerica: a disease primarily driven by occupational heat stress. Revista Panamamericana de Salud Pública. 44 e15. https://doi.org/10.26633/RPSP.2020.15

Zuñiga, C., Müller, H., \& Flores, M. (2011). Prevalencia de enfermedad renal crónica en centros urbanos de atención primaria. Revista Médica de Chile, 139(9), 1176-1184. https://doi.org/10.4067/ S0034-98872011000900010 